\title{
Factor VIII-Bypassing Activity of Bovine Tissue Factor Using the Canine Hemophilic Model
}

\author{
Donogh P. O'Brien, Alan R. Giles, * Keri M. Tate, and Gordon A. Vehar \\ Department of Cardiovascular Research, Genentech, Inc., South San Francisco, California 94080; and *Departments of Pathology and \\ Medicine, Queen's University, Kingston, Ontario, Canada
}

\begin{abstract}
The bleeding disorder of hemophilia A currently treated by replacement therapy of the missing coagulation factor, factor VIII, is frequently complicated by the development of neutralixing antibodies. The therapeutic potential of attenuated forms of the lipid-associated glycoprotein tissue factor, a known initiator of coagulation, was investigated as a factor VIII-bypassing activity. The protein moiety of tissue factor (Apo-TF) was partially purified and exhibited minimal procoagulant activity before relipidation in vitro. In pilot studies, Apo-TF injection into rabbits previously anticoagulated with an antibody to factor VIII was found to have a procoagulant effect. The efficacy of the material was further demonstrated when injection of Apo-TF in hemophilic dogs resulted in a normalization of the cuticle bleeding time. Little or no change in the blood parameters associated with disseminated intravascular coagulation was observed at lower doses, although mild to moderate effects were seen at higher doses. These data suggest a novel role for Apo-TF preparations as a potential therapeutic agent for hemophiliacs with antibodies to factor VIII once the potential thrombogenicity of such materials is evaluated.
\end{abstract}

\section{Introduction}

Hemophilia A (classic hemophilia) is one of the most common inherited disorders of blood coagulation. Afflicted individuals experience bleeding episodes owing to a deficiency of factor VIII, a cofactor that functions in the middle of the coagulation cascade (1). Treatment of hemophilia currently involves replacement therapy using blood-derived concentrates of factor VIII.

As a result of the repeated injections of factor VIII-containing preparations, $\sim 15 \%$ of all patients with hemophilia $\mathrm{A}$ develop neutralizing antibodies (also referred to as inhibitors) against factor VIII (for a review, see reference 2). As a result, replacement therapy using factor VIII preparations becomes ineffective as a means of correcting bleeding episodes. A variety of methods have been utilized to treat bleeding episodes in inhibitor patients, including immunosuppression (3), antibody depletion through plasmapheresis (4), high-dose factor VIII therapy leading to immune tolerance (5-7), and repeated treatment with factor VIII combined with immunosuppression (8). Prothrombin complex concentrates have proven to be

Address reprint requests to Dr. Vehar.

Received for publication 2 September 1987 and in revised form 20 January 1988.

J. Clin. Invest.

(c) The American Society for Clinical Investigation, Inc.

0021-9738/88/07/0206/06 \$2.00

Volume 82, July 1988, 206-211 partially effective (9-11) but their use is controversial owing to uncertainty with regard to the putative factors responsible (12-14), thrombogenicity (15-17), and the risk of viral infections. One component of prothrombin complex concentrates, factor VIIa, has been reported to be efficacious in the treatment of homophiliacs with inhibitors (18). Highly purified porcine factor VIII is also effective in some patients (19).

Tissue factor (TF) ${ }^{1}$ is a potent thromboembolic agent in vivo. This lipid-associated cofactor, in complex with factor VIIa, accelerates the conversion of factors IX and X to IXa and $\mathrm{Xa}$, respectively (1). As such, TF results in clot formation by a mechanism that bypasses the factor VIII step in the coagulation cascade. Numerous investigators have demonstrated that crude TF from tissue homogenates induces disseminated intravascular coagulation when infused at high concentrations. Because of the potent coagulant activity of these preparations, TF has not been seriously considered as a therapeutic agent. Recent work, however, has shown that the delipidated protein moiety of TF (Apo-TF) is inactive, requiring relipidation in order to exhibit any in vitro coagulant activity (for a review, see reference 20 ). We decided to investigate the in vivo coagulant effects of Apo-TF preparations. Apo-TF shortened the bleeding time in preliminary experiments employing a hemophilic rabbit model. This pilot study precipitated a more rigorous test of Apo-TF as potential factor VIII bypassing activity in hemophilic dogs (21). These data suggest the possible utility of Apo-TF as a treatment for patients with hemophilia and inhibiting antibodies.

\section{Methods}

Materials. Mature bovine brains were obtained from Pel-Freeze Biologicals, Rogers, AR. Triton X-100 (TX-100) and $\alpha$-D-methylglucoside were from Calbiochem-Behring Corp., San Diego, CA. Concanavalin A-Sepharose was from Pharmacia Fine Chemicals, Piscataway, NJ. All other chemicals were from Sigma Chemical Co., St. Louis, MO and were reagent grade or better. Coagulation factor-deficient and normal pooled human plasmas were from George King Biomedical, Overland Park, KS. Bovine factor VII was purified by the method of Broze et al. (22). Bovine factor $X$ was obtained from Enzyme Research Labs, Inc., South Bend, IN. Chromogenic substrate S2222/12581 was obtained from Helena Laboratories, Beaumont, TX.

Measurement of blood parameters. Blood for coagulation was anticoagulated with sodium citrate (3.8\% wt/vol), $9 \mathrm{vol}$ of blood to $1 \mathrm{vol}$ of anticoagulant. The prothrombin time and activated partial thromboplastin time assays were measured on a Coagamate 2001 using General Diagnostics (Morris Plains, NJ) reagents as described previously (21). Factor VIII and factor V levels in dogs were measured using one-stage assays with congenitally deficient plasmas as previously described (23). Rabbit factor VIII was measured by chromogenic assay (Coatest, Helena Laboratories). In order to determine cross-reaction of human

1. Abbreviations used in this paper: CBT, cuticle bleeding time; TF, tissue factor. 
anti-factor VIII antibody with rabbit factor VIII, rabbit plasma was substituted for human plasma in the incubation mixture. Platelets were measured on a Coulter counter (Coulter Electronics, Hialeah, FL). Fibrinogen values were measured using the Clauss procedure (24). Control canine plasma was obtained by pooling plasma from 20 normal dogs.

Relipidation of $T F$. Detergent-solubilized TF has minimal procoagulant activity prior to relipidation. The relipidation mixture was prepared by adding $50 \mu \mathrm{l}$ of a $5 \mathrm{mg} / \mathrm{ml}$ solution of phosphatidylcholine (lecithin) in $0.25 \%$ deoxycholic acid and $25 \mu \mathrm{l}$ of $100 \mathrm{mM} \mathrm{CdCl}_{2}$ to 0.8 $\mathrm{ml}$ of TBSA (consisting of $0.05 \mathrm{M}$ Tris, $0.1 \mathrm{M} \mathrm{NaCl}, 0.1 \% \mathrm{BSA}, \mathrm{pH}$ 7.5). Between 1 and $10 \mu \mathrm{l}$ of the solution to be assayed was added and the mixture incubated for $30 \mathrm{~min}$ at $37^{\circ} \mathrm{C}$.

Chromogenic TF assay. Relipidated TF was diluted at least 1:10 in TBSA and $10 \mu$ l transferred to a $12 \times 75$ polystyrene test tube. $50 \mu \mathrm{l}$ of a solution containing bovine factor $\mathrm{X}(0.127 \mu \mathrm{g})$ and factor $\mathrm{VII}_{\mathrm{a}}(4 \mathrm{ng})$ were then added with $100 \mu \mathrm{l}$ of $25 \mathrm{mM} \mathrm{CaCl}_{2}$. Tubes were incubated at $37^{\circ} \mathrm{C}$ for $5 \mathrm{~min}$ and $50 \mu \mathrm{l} \mathrm{S} 2222 / \mathrm{I} 2581$ chromogenic substrate was added. Incubation for a further $10 \mathrm{~min}$ at $37^{\circ} \mathrm{C}$ was stopped by the addition of $100 \mu \mathrm{l}$ of $50 \%$ glacial acetic acid. Absorbance was detected at $405 \mathrm{~nm}$. A standard curve was constructed using rabbit brain thromboplastin (Sigma Chemical Co.) reconstituted according to the manufacturer's instructions. One unit of TF activity was defined as that contained in $10 \mu \mathrm{l}$ of this material.

One-stage assay for TF. Relipidated TF was diluted at least 1:10 in TBSA and $50 \mu \mathrm{l}$ was transferred to a $10 \times 75-\mathrm{mm}$ siliconized glass test tube. $100 \mu \mathrm{l}$ of pooled normal human plasma were then added. The tubes were warmed for $1 \mathrm{~min}$ at $37^{\circ} \mathrm{C}$ and $100 \mu \mathrm{l}$ of $25 \mathrm{mM} \mathrm{CaCl}_{2}$ was added. Clot formation was timed and all samples were assayed in duplicate.

Purification of bovine brain TF. Bovine brain TF was partially purified by a modification of the method of Bach et al. (25). TF activity was followed using the chromogenic assay. Bovine brains were acetone delipidated, detergent extracted, and affinity purified on concanavalin A-Sepharose as described (25). The eluate from the concanavalin ASepharose column was dialyzed against $0.05 \mathrm{M}$ Tris- $\mathrm{HCl}, 0.10 \mathrm{M}$ $\mathrm{NaCl}$, pH 7.4 (TBS) containing $0.1 \% \mathrm{TX}-100$ for $18 \mathrm{~h}$. The eluate was then concentrated to a volume of $20 \mathrm{ml}$ in an Amicon Corp. (Danvers, MA) stirred cell with a YM-30 membrane. This material was applied to a $120 \times 1.5-\mathrm{cm}$ column of AcA-44 Ultrogel resin (LKB Instruments, Gaithersburg, MD), preequilibrated with TBS $0.1 \%$ TX-100. The column was developed isocratically at a flow rate of $6 \mathrm{ml} / \mathrm{h}$. 1-ml fractions were collected and aliquots relipidated for assay. Peak activity fractions were pooled and stored at $-20^{\circ} \mathrm{C}$. We estimate this material to be $\sim 10 \%$ pure.

Demonstration of in vivo factor VIII-bypassing activity of TF. A model for hemophilia A with inhibitors was developed by infusing normal rabbits with a cross-reacting human anti-factor VIII antibody. Arterial and venous cannulae were inserted into the ears of restrained New Zealand White rabbits ( $1.8 \mathrm{~kg})$. Arterial blood was withdrawn and anticoagulated for assay. The first $1 \mathrm{ml}$ of blood was discarded. Blood was kept on ice before centrifugation to collect platelet-poor plasma. Each animal then received $600 \mu \mathrm{l}$ of antibody, 1,700 Bethesda $U / \mathrm{ml}$, via venous cannulae. $30 \mathrm{~min}$ after infusion blood samples were withdrawn for assay. The control animal received an infusion of $300 \mu \mathrm{l}$ of TBS $0.1 \%$ TX -100 through the venous cannula. The test animal received the same volume of buffer containing purified TF apoprotein. On relipidation, this represented a dose of $233 \mathrm{U} / \mathrm{kg}$ as measured in the chromogenic assay. $60 \mathrm{~min}$ after the infusion of antibody, arterial cannulae were removed and blood flow volume and duration were recorded.

Demonstration of factor VIII-bypassing activity in a canine hemophilia model. In vivo factor VIII-bypassing activity was measured in hemophilic dogs utilizing the cuticle bleeding time assay (CBT) (21). Fasting animals were anesthetized and a CBT performed before any infusion. Lack of Apo-TF toxicity was first determined in a normal dog on bolus injection of $50 \mathrm{Apo}-\mathrm{TF} \mathrm{U} / \mathrm{kg}$ and $250 \mathrm{Apo}-\mathrm{TF} \mathrm{U} / \mathrm{kg}$ doses. CBTs were performed prior to infusion and $30 \mathrm{~min}$ after each injection. Blood was withdrawn for coagulation assays at various time points during the experiment. In order to demonstrate in vivo factor VIII-bypassing activity of Apo-TF, experiments were conducted using hemophilic dogs. Apo-TF was administered by bolus injection and CBTs performed at various time points up to $90 \mathrm{~min}$ after the infusion. Several doses were chosen ranging from 50 to $250 \mathrm{Apo}-\mathrm{TF} \mathrm{U} / \mathrm{kg}$. Blood samples were withdrawn throughout the duration of each experiment and assayed for factor $\mathrm{V}$ and prothrombin and partial thromboplastin times. CBTs of greater than 12 min were regarded as grossly abnormal and thus, nails were cauterized to prevent excessive blood loss.

\section{Results}

Tissue factor was partially purified ( $\sim 12,000$-fold) from acetone brain powders (Table I) and subsequently assayed in both a modified prothrombin time assay and a chromogenic TF assay utilizing purified coagulation factors (Table II). In both assays the Apo-TF preparation exhibited minimal procoagulant activity before relipidation. The procoagulant activities of relipidated TF were dependent on the presence of factor VII in both assays (data not shown).

In order to observe the action of Apo-TF in vivo, a rabbit hemophilic model was developed in which normal rabbits were first infused with a high-titer cross-reacting human antibody to factor VIII. By utilizing this antibody, it was possible to reduce rabbit factor VIII levels from $\sim 5 \mathrm{U} / \mathrm{ml}$, as assayed using a pooled human standard, to undetectable levels within 30 min (Table III). Both duration and volume of blood loss from the artery of the ear after removal of the cannulae were dramatically reduced in the animal receiving Apo-TF compared with the animal receiving a buffer control (Table III). These results suggested that Apo-TF could be well tolerated

Table I. Purification of Bovine Brain Tissue Factor

\begin{tabular}{|c|c|c|c|c|c|c|c|}
\hline \multirow[b]{2}{*}{ Sample } & \multirow[b]{2}{*}{ Volume } & \multicolumn{2}{|c|}{ Protein } & \multicolumn{2}{|c|}{ TF activity } & \multirow[b]{2}{*}{ Specific activity } & \multirow[b]{2}{*}{ Purification } \\
\hline & & Concn. & Total & & Total units & & \\
\hline & $m l$ & $m g / m l$ & $m g$ & $U / m l$ & & $U / m g$ & Fold \\
\hline Acetone brain powders & 3,500 & 7.35 & 25,725 & 1.06 & 3,675 & 0.14 & - \\
\hline TBS wash supernatant & 3,000 & 6.04 & 18,120 & 0.16 & 480 & - & - \\
\hline $0.1 \%$ Triton supernatant & 3,000 & 1.42 & 4,260 & 0.52 & 1,560 & - & - \\
\hline $2 \%$ Triton extract & 2,750 & 3.00 & 8,250 & 14.82 & 40,761 & 4.94 & 35.2 \\
\hline Concanavalin A-Sepharose eluate & 420 & 0.17 & 71 & 53.50 & 22,470 & 314.00 & 2242.0 \\
\hline Ultrogel AcA-44 pool & 8 & 0.83 & 6 & 1400.00 & 10,780 & $1,711.00$ & $12,221.0$ \\
\hline
\end{tabular}




\begin{tabular}{|c|c|c|c|c|c|}
\hline \multirow[b]{2}{*}{ Sample } & \multirow[b]{2}{*}{ Dilution } & \multicolumn{2}{|c|}{ Prothrombin time } & \multirow{2}{*}{$\begin{array}{l}\text { Chromogenic assay } \\
\qquad A 405 \mathrm{~nm}\end{array}$} & \multirow[b]{2}{*}{ TF } \\
\hline & & \multicolumn{2}{|c|}{ Clot time } & & \\
\hline & & $s$ & $s$ & & $U / m l$ \\
\hline \multirow[t]{2}{*}{ Phosphatidylcholine/deoxycholate relipidation mixture } & 1:10 & 120.6 & 121.4 & 0.000 & 0.0 \\
\hline & $1: 100$ & 120.7 & 121.4 & 0.000 & 0.0 \\
\hline \multirow[t]{3}{*}{ Relipidated TF } & 1:10 & 22.1 & 22.6 & $>2.0$ & ND \\
\hline & $1: 100$ & 58.1 & 58.7 & $>2.0$ & ND \\
\hline & 1:1,000 & ND & ND & 1.493 & 6,967 \\
\hline \multirow[t]{2}{*}{ Apo-TF } & 1:10 & 117.1 & 116.2 & 0.047 & 0.0 \\
\hline & $1: 100$ & 118.4 & 118.7 & 0.093 & 0.0 \\
\hline
\end{tabular}

Phospholipid dependence of purified Apo-TF procoagulant activity was measured by a modified prothrombin time (wherein the designated sample was substituted for thromboplastin) and chromogenic assay using purified coagulation factors. ND, values either not determined or not calculable.

and might correct the bleeding diathesis associated with factor VIII inhibitors. In order to confirm this observation in a more relevant model, the safety and efficacy of Apo-TF was tested in normal and hemophilic dogs.

An anesthetized normal dog was administered doses of Apo-TF representing 50 and 250 Apo-TF U/kg on relipidation in the chromogenic assay. The CBT in this animal was $\sim 3$ min prior to any infusion (Fig. 1). Factor $\mathrm{V}$ levels were normal 30 min after each infusion (Table IV). The prothrombin and partial thromboplastin times were unchanged at the end of the experiment and the CBTs were also within the normal range. The fibrinogen level was unchanged at the lower dose and dropped slightly at the higher dose (Table IV). Thus infusion of Apo-TF was reasonably well tolerated in normal dogs.

A hemophilic dog with a prolonged CBT characteristic of hemophilia A was administered $50 \mathrm{U} / \mathrm{kg}$ of Apo-TF. The CBT was normalized $30 \mathrm{~min}$ after this infusion (Fig. 1). This correction was not associated with an alteration in factor $\mathrm{V}$ levels and the prothrombin time was not lengthened, although there was a slight decrease in the fibrinogen level (Table IV). The procoagulant effect was not maintained $90 \mathrm{~min}$ after the infusion as the CBT was again abnormal at this time point. A dose-response relationship was established by infusion of $250 \mathrm{Apo}-\mathrm{TF}$ $\mathrm{U} / \mathrm{kg}$. At this dose, the CBT of the hemophilic dog was normalized at 30 and 90 min (Fig. 1). This increased dosage was, however, associated with a decrease in factor $\mathrm{V}$ levels, a slight lengthening of the prothrombin time, and a $50 \%$ drop in fibrinogen (Table IV).

Experiments were repeated using a dose of 100 Apo-TF $\mathrm{U} / \mathrm{kg}$ in order to obtain the maximum duration of efficacy while further evaluating the effect on blood parameters. A hemophilic dog received 100 Apo-TF U/kg and CBTs performed at 15, 30, and $45 \mathrm{~min}$. Interestingly, the CBT at $15 \mathrm{~min}$ was still abnormal (Fig. 1) with stasis achieved at 30 min after the infusion. This is an observation consistent with results obtained using conventional canine factor VIII preparations in noninhibitor hemophilic dogs (A. R. Giles, unpublished observation). At this dose, the CBT was normal at $45 \mathrm{~min}$. Blood samples were taken and analyzed for evidence of consumptive coagulopathy (Table IV). Factor V levels, prothrombin times, and platelet and fibrinogen levels showed little or no change. Thus, efficacy of Apo-TF in vivo was demonstrated at a dose that did not appear to cause complication arising from disseminated intravascular coagulation.

The factor VIII-bypassing activity was confirmed in a third hemophilic dog using a dose of 100 Apo-TF U/kg and CBTs performed at 30 and $45 \mathrm{~min}$. While efficacy was established at both time points, some rebleeding occurred at $45 \mathrm{~min}$.

\section{Discussion}

The appearance of a neutralizing antibody to factor VIII in a patient with hemophilia makes treatment of a bleeding episode extremely difficult. One therapeutic regimen that has proven somewhat effective as a factor VIII bypassing activity is the use of activated prothrombin complex concentrates. Although controversial, the positive therapeutic effect may result from the thrombogenic potential of these concentrates caused by the presence of activated coagulation proteins (thrombin, VIIa, IXa, and Xa) (12-14). TF has long been recognized as a potent

Table III. Demonstration of Factor VIII Bypassing Activity of Apo-TF In Vivo after Injection of Human Anti-Factor VIII Antibody in Rabbits

\begin{tabular}{|c|c|c|c|c|c|c|c|}
\hline \multirow[b]{3}{*}{ No. } & \multirow[b]{3}{*}{ Weight } & \multicolumn{2}{|c|}{ Factor VIII } & \multirow{2}{*}{\multicolumn{2}{|c|}{ Apo-TF injection }} & \multirow{2}{*}{\multicolumn{2}{|c|}{ Arterial bleeding }} \\
\hline & & \multirow[b]{2}{*}{ Before } & \multirow{2}{*}{$\begin{array}{l}30 \text { min after } \\
\text { sample injection }\end{array}$} & & & & \\
\hline & & & & U/Total & $\mathbf{U} / \mathbf{k g}$ & Duration & Volume \\
\hline & kg & & & & & $\min$ & $m l$ \\
\hline 1 & 1.85 & 5.0 & 0.0 & 0 & 0 & 11.00 & 15.200 \\
\hline 2 & 1.80 & 4.8 & 0.0 & 420 & 233 & 0.63 & 0.125 \\
\hline
\end{tabular}




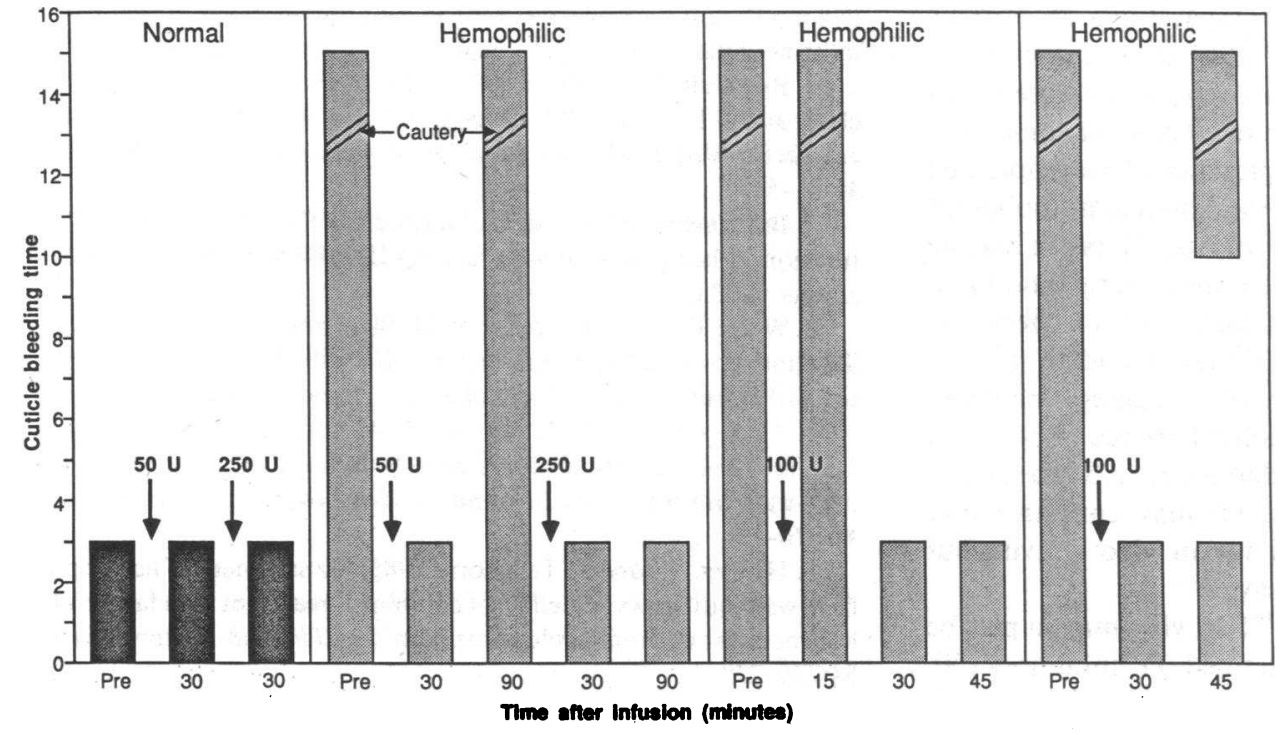

Figure 1. Cuticle bleeding times in animals receiving Apo-TF. Arrows denote dose of Apo-TF in U/kg administered at time zero. Pre refers to CBT prior to any injection. The left panel represents the CBT of a normal dog before and after two infusions of Apo-TF. The remaining panels represent the data obtained after infusion of Apo-TF into three separate hemophilic dogs. thrombogenic agent in vivo, and numerous investigators have utilized crude preparations of this membrane-bound glycoprotein to induce disseminated intravascular coagulation in animals. We have performed experiments to determine whether a partially purified, attenuated form of TF can bypass factor VIII activity in vivo.

The observation that the components of the extrinsic pathway (TF-factor VII) are able to form a reinforcement loop with components of the intrinsic pathway (factors VIII, IX, and $X$ ) suggests a dominant role of the extrinsic pathway in the initiation of the coagulation cascade $(26,27)$. In this regard, it is interesting to note that at low concentrations of TF, the prothrombin time of hemophilic plasma is prolonged, a phenomenon corrected by the addition of higher concentrations of TF (28). Clearly, the potential for further direct activation of factor $\mathrm{X}$ by the extrinsic complex exists in hemophilic plasma, a concept supported by the efficacy of factor VIIa infusions in the treatment of bleeding episodes of hemophiliacs with inhibitors (18).

Pilot studies in rabbits strongly suggested that while the apoprotein had little activity in in vitro assay systems, it was capable of affecting the bleeding induced in an animal infused with anti-factor VIII antibodies. We subsequently confirmed these results in normal and hemophilic dogs by measurement of the CBT (21); Apo-TF resulted in the CBT correction of three separate animals. Little or no change in blood parameters associated with disseminated intravascular coagulation was observed at lower doses, although mild to moderate effects were seen at higher doses. Although the efficacy may be transient in nature, the data suggest that Apo-TF may be a promising new therapeutic agent for the treatment of hemophiliacs having factor VIII antibodies. Obviously, a detailed study of possible adverse effects (e.g., disseminated intravascular coagulation) will be required using purified human tissue factor.

Table IV. Blood Parameters in Normal and Hemophilic Dogs after Injection of Apo-TF

\begin{tabular}{|c|c|c|c|c|c|c|c|}
\hline Dog & $\begin{array}{l}\text { Dose } \\
\text { Apo-TF }\end{array}$ & $\begin{array}{l}\text { Sample time } \\
\text { after injection }\end{array}$ & PT & PTT & Factor $\mathrm{V}$ & Platelets & Fibrinogen \\
\hline & $U / k g$ & $\min$ & & & $U / m l$ & $10^{6} / \mathrm{ml}$ & g/liter \\
\hline \multirow[t]{4}{*}{$N$} & 50 & PRE & 12 & 21 & 0.81 & ND & 2.48 \\
\hline & & 67 & 12 & 22 & 0.96 & ND & 2.51 \\
\hline & 250 & 30 & 12 & 19 & 1.07 & ND & 2.79 \\
\hline & & 60 & 12 & 16 & 1.22 & ND & 2.31 \\
\hline \multirow[t]{3}{*}{ H1 } & 50 & PRE & 13 & 53 & 1.01 & ND & 1.54 \\
\hline & & 150 & 13 & 54 & 1.03 & ND & 1.26 \\
\hline & 250 & 32 & 15 & 71 & 0.64 & ND & 0.77 \\
\hline \multirow[t]{3}{*}{$\mathrm{H} 2$} & 100 & PRE & 13 & 51 & 1.24 & 205 & 1.24 \\
\hline & & 15 & 13 & 51 & 1.23 & 169 & 1.15 \\
\hline & & 57 & 13 & 51 & 1.17 & 223 & 1.09 \\
\hline
\end{tabular}

Coagulation assay results after bolus injection of Apo-TF in normal and hemophilic dogs. N, normal dog; H1 and H2, hemophilic dogs; ND, not determined. PT, prothrombin time; PTT, partial thromboplastin time; PRE, sample taken prior to injection. Values for PT and PTT are mean of duplicate determinations. Factor $\mathrm{V}$ levels are mean of four assays. Fibrinogen values are the mean values obtained at two dilutions. 
The mechanism whereby these preparations of partially purified Apo-TF are exerting this effect is unknown. It is known that Apo-TF has cofactor activity in the absence of phospholipid, albeit with a catalytic rate constant some 160fold lower than that achieved in the presence of the relipidated molecule (29). Furthermore, Apo-TF will bind to immobilized factor VII $(22,29)$. Thus the injection of Apo-TF per se may be sufficient to bypass the factor VIII requirement by binding to factor VII and producing sufficient factor $\mathrm{Xa}$ to effect clot formation. Alternatively, the Apo-TF may relipidate in vivo, possibly at the site of vascular injury where aggregating platelets could act as a suitable phospholipid source. Apo-TF is nonfunctional without prior relipidation in in vitro assays. However, our preparation of Apo-TF may contain small amounts of phospholipid sufficient for in vivo activity but which is undetectable by in vitro assays.

The relatively transient effect of TF in vivo may in part be due to the presence of recently described TF inhibitors (30, 31). This mechanism of neutralization is apparently not sufficient to prevent the factor VIII-bypassing activity of Apo-TF in vivo, possibly due to a lack of recognition of the delipidated form of tissue factor.

Treatment of patients with hemophilia A who have neutralizing antibodies is extremely difficult. In many cases, concentrate therapy is restricted to organ-threatening or lifethreatening bleeding in these patients. Activated prothrombin concentrates have shown efficacy, but are expensive, less active than replacement therapy with factor VIII, and can cause disseminated intravascular coagulation (15-17). The active component(s) of these preparations is still a matter of controversy (12-14), although originally proposed to be activated products of the clotting factors II, VII, IX, and X (32). In any event, there is a great need for a truly effective alternative means of treatment of these individuals. For this reason, the results reported in this study are highly encouraging, suggesting that homogeneous preparations in the microgram range would be efficacious in humans.

In conclusion, these results suggest that an attenuated form of bovine TF may be a new potential therapeutic agent. Trace quantities of the purified bovine protein were found to effect a transient correction of the bleeding diathesis in hemophilic dogs. With the obvious qualifiers of cross-species testing of impure material and the potential for adverse systemic effects, the potential exists that purified human tissue factor could be developed into an efficacious therapeutic agent.

\section{Acknowledgments}

The excellent technical assistance of James McCabe, Hugh Hoogendorn, and Shawn Tinlin is gratefully acknowledged.

This work was supported in part by a grant from the Medical Research Council of Canada (MA-7667).

\section{References}

1. Jackson, C. M., and Y. A. Nemerson. 1980. Blood coagulation. Annu. Rev. Biochem. 49:765-811.

2. Hoyer, L. W., editor. 1984. Factor VIII Inhibitors. Alan R. Liss, Inc., New York. 420 pp.

3. Hruby, M. A., and I. Schulman. 1973. Failure of combined factor VIII and cyclophosphamide to suppress antibody to factor VIII in hemophilia. Blood. 42:913-923.

4. Regnault, V., C. Rivat, J-P. Vallet, C. Francois-Gerard, G. Brochier, and J. P. Allain. 1987. A potential new procedure for removing anti-factor VIII antibodies from hemophilic plasma. Thromb. Res. 45:51-57.

5. Brackmann, H. H., and I. Gormsen. 1977. Massive factor VIII infusion in haemophiliac with factor VIII inhibitors, high responder. Lancet. ii.933.

6. White, G. C., R. E. Taylor, P. M. Blatt, and H. R. Roberts. 1983. Treatment of a high titer anti-factor VIII antibody by continuous factor VIII administration: report of a case. Blood. 62:141-145.

7. Gomperts, E. D., S. Jordan, J. A. Church, R. Sakai, and J. Lemire. 1984. Induction of tolerance to factor VIII in a child with a high-titer inhibitor: in vitro and in vivo observations. J. Pediatr. 104:70-75.

8. Hedner, V., and L. Tengborn. 1985. Management of haemophilia A with antibodies: the effect of combined treatment with factor VIII hydrocortisone and cyclophosphamide. Thromb. Haemostasis. 54:776-779.

9. Lusher, J. M., S. S. Shapiro, J. E. Palascak, A. V. Rao, P. H. Levine, P. M. Blatt, and the Hemophilia Study Group. 1980. Efficacy of prothrombin complex concentrates in hemophiliacs with antibodies to factor VIII. N. Engl. J. Med. 303:421-425.

10. Sjamsoedin, L., L. Heijnen, E. P. Mausen-Bunschoten, J. L. van Geijlswijk, H. van Houwelingen, P. van Asten, and J. J. Sixma. 1981. The effect of activated prothrombin complex concentrate (FEIBA) on joint and muscle bleeding in patients with hemophilia $A$ and antibodies to factor VIII: A double-blind clinical trial. N. Engl. J. Med. 305:717-721.

11. Lusher, J. M., P. M. Blatt, J. A. Penner, L. M. Aledort, P. H. Levine, C. G. White, A. I. Warrier, and D. A. Whitehurst. 1983. Autoplex versus proplex: a controlled, double-blind study of effectiveness in acute hemarthrosis in hemophilics with inhibitors to factor VIII. Blood. 62:1135-1138.

12. Roberts, H. 1981. Hemophiliacs with inhibitors: therapeutic options. N. Engl. J. Med. 305:757-758.

13. Aronson, D. L. 1984. There is no correlation between vitamin $\mathrm{K}$ dependent coagulant content and clinical effectiveness of activated and standard factor IX complex concentrates. In Factor VIII Inhibitors. L. W. Hoyer, editor. Alan R. Liss, Inc., New York. 243-250.

14. Giles, A. R. 1987. By what mechanisms could prothrombin complex concentrates promote factor VIII bypassing activity in vivo? Transf. Med. Rev. 1:131-137.

15. Cederbaum, A. I., P. M. Blatt, and H. Roberts. 1976. Intravascular coagulation with use of human prothrombin complex concentrates. Ann. Intern. Med. 84:683-687.

16. Fukui, H., Y. Fujimura, Y. Takahashi, S. Mikami, and A. Yoshioka. 1981. Laboratory evidence of DIC under FEIBA treatment of a hemophilic patient with intracranial bleeding and high titre factor VIII inhibitor. Thromb. Res. 22:177-184.

17. Abildgaard, C. F. 1981. Hazards of prothrombin-complex concentrates in treatment of hemophilia. N. Engl. J. Med. 304:670-71.

18. Hedner, U., and W. Kisiel. 1983. Use of human factor VIIa in the treatment of two hemophilia A patients with high-titer inhibitors. J. Clin. Invest. 71:1836-1841.

19. Gatti, L., P. M. Mannucci. 1984. Use of procine factor VIII in the management of seventeen patients with factor VIII antibodies. Thromb. Haemostasis. 51:379-384.

20. Nemerson, Y., and R. Bach. 1982. Tissue factor revisited. Prog. Hemostasis Thromb. 6:237-261.

21. Giles, A., Tinlin, S., and Greenwood, R. 1982. A canine model of hemophilic (factor VIII:C deficiency) bleeding. Blood. 60:727-730.

22. Broze, G. J., J. E. Leykam, B. D. Schwartz, and J. P. Miletich. 1985. Purification of human brain tissue factor. J. Biol. Chem. 260:10917-10920. 
23. Giles, A. R., M. E. Nesheim, and K. G. Mann. 1984. Studies of factors V and VIII:C in an animal model of disseminated intravascular coagulation. J. Clin. Invest. 74:2219-2225.

24. Clauss, V. A. 1957. Gerinnungs schnellmethode zur bestmung des fibrinogens. Acta Haematol. 17:237-246.

25. Bach, R., Y. Nemerson, and W. Konigsberg. 1981. Purification and characterization of bovine tissue factor. J. Biol. Chem. 256:8324.

26. Osterud, B., and S. Rapaport. 1977. Activation of factor IX by the reaction product of tissue factor and factor VII: additional pathway for initiating blood coagulation. Proc. Natl. Acad. Sci. USA. 74:52605264.

27. Nemerson, Y. 1983. Regulation of initiation of coagulation by factor VII. Haemostasis. 13:150-155.

28. Rapaport, S. I. 1981. The activation of factor IX by the tissue factor pathway. In Hemophilia and Hemostasis. D. Menache, D. Sur- genor, N. Mac, and H. Anderson, editors. Alan R. Liss, Inc., New York. 57-76.

29. Ghua, A., R. Bach, W. Konigsberg, and Y. Nemerson. 1986. Affinity purification of human tissue factor: interaction of factor VII and tissue factor in detergent micelles. Proc. Natl. Acad. Sci. USA. 83:299-302.

30. Sanders, N. L., S. P. Bajaj, A. Zivellin, and S. I. Rapaport. 1985. Inhibition of tissue factor/factor VIIa activity in plasma requires factor $\mathrm{X}$ and an additional plasma component. Blood. 66:204-212.

31. Carson, S. D. 1981. Plasma high density lipoproteins inhibit the activation of coagulation factor $\mathrm{X}$ by factor VIIa and tissue factor. FEBS (Fed. Eur. Biochem. Soc.) Lett. 132:37-40.

32. Breen, F. A., and J. L. Tullis. 1969. Prothrombin concentrates in treatment of Christmas disease and allied disorders. JAMA (J. Am. Med. Assoc.) 208:1848-1852. 